\title{
LXIII.-A Synthesis of Tropinone.
}

\section{By Robert Robinson.}

AMONG the noteworthy achievements of Willstätter in connexion with his investigation of the chemistry of tropine, there was nothing that contributed a greater share to the final solution of the problems encountered than the demonstration of the constitution of tropinone, a ketone first produced in 1896 (Willstätter, Ber., 1896, 29, 396; Ciamician and Silber, ibid., 490) by the oxidation of tropine, and later by a similar method from ecgonine (Willstätter, Ber., 1898, 31, 2655). This substance has become the central figure in the atropine group of the alkaloids, and, as shown below, may be regarded as the natural starting point in the synthetical preparation of a number of bases of great value in the practice of medicine and surgery. 
ROBINSON : A SYNTHESIS OF TROPINONE. View $763^{2}$

Tropine $\underset{\text { reduction }}{\stackrel{\text { electrolytic }}{\leftarrow}}$ Tropinone $\underset{\mathrm{CO}_{2}}{\stackrel{\mathrm{Na}}{\longrightarrow}}$ Tropinonecarboxylic acid

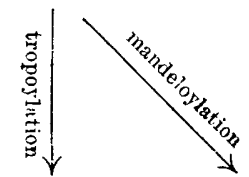

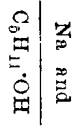

$$
\begin{aligned}
& \psi \text {-Tropine }
\end{aligned}
$$

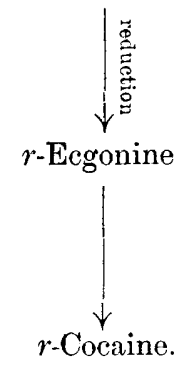

An examination of the details of the reactions involved in the above shows that it is improbable that cocaine would be synthesised from artificial tropinone, and that the feasibility of the preparation of atropine would depend on the elaboration of improved methods for the production of tropic acid. On the other hand, given readily accessible tropinone, the technical preparation of homatropine and tropacocaine would be a comparatively simple matter. By reason of the richness of the field of chemistry explored, the synthesis of tropine by Willstätter (Annalen, 1901, 317, 204; 1903, 326, 1) is classical, but the method employed, which it is unnecessary to recall in all its details, appears to be of too complicated a character to admit of development into an economical process. It is first of all necessary to obtain suberone, which is then converted through several stages into cycloheptatriene, and finally into tropidine, $\psi$-tropine, tropinone, and tropine.

Nevertheless, an inspection of the formula of tropinone (I) discloses a degree of symmetry and an architecture which justify the hope that the base may ultimately be obtained in good yield as the product of some simple reaction and from accessible materials. By imaginary hydrolysis at the points indicated by the dotted lines, the substance may be resolved into succindialdehyde, methylamine, and acetone, and this observation suggested a line of attack of the problem which has resulted in a direct synthesis.

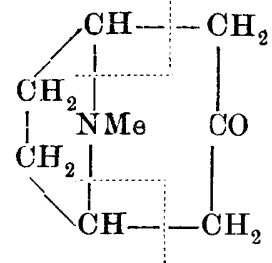

(I.)

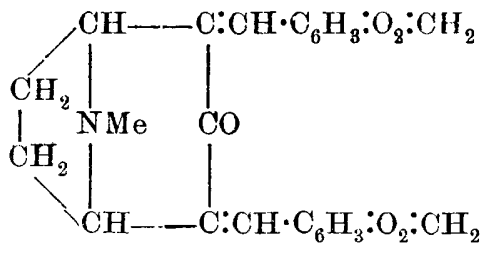

(II.) 
In searching for a method of synthesis of any substance, it is always convenient to be able to recognise the formation of traces of the desired product, and in the present instance none of the derivatives of tropinone already prepared was found to be so suitable for its detection as the dipiperonylidene derivative (II), which does not appear to have been hitherto described. With the aid of this highly characteristic compound it was proved that tropinone is obtained in small yield by the condensation of succindialdehyde with acetone and methylamine in aqueous solution. An improvement followed on the replacement of the acetone by a salt of acetonedicarboxylic acid. The initial product is a salt of tropinonedicarboxylic acid, and this loses two molecules of carbon dioxide with the formation of tropinone when the solution is acidified and heated.

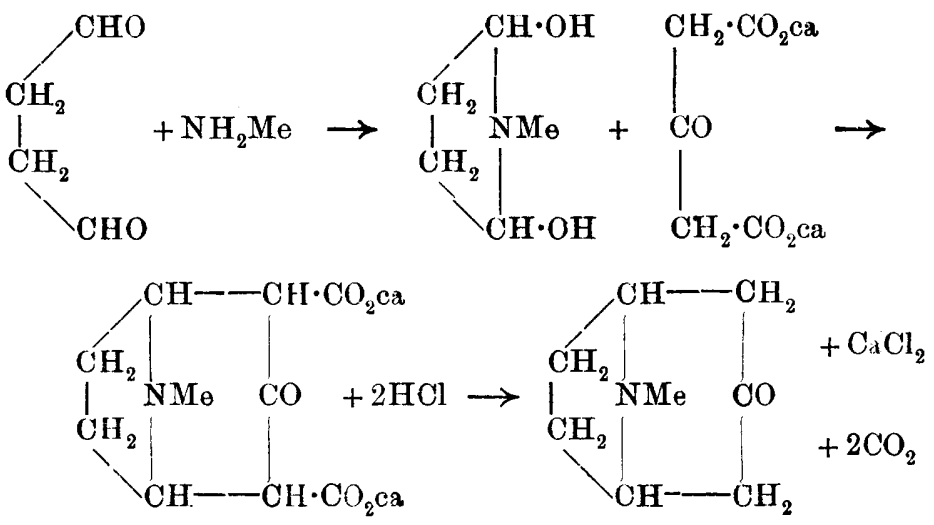

Tropinone was also obtained by the hydrolysis of the product of condensation of succindialdehyde with ethyl acetonedicarboxylate and methylamine in alcoholic solution. When more material is available it is hoped to institute a series of experiments with the object of defining the optimum conditions of the synthesis, and it will then be possible to state with more exactness the maximum yield obtainable. However, in spite of the small experience so far gained, an experiment is described in which the yield of dipiperonylidenetropinone from succindialdehyde was 42 per cent. of that theoretically possible. The method of synthesis of bicyclic bases which has been developed is probably of general application, and an attempt will be made to synthesise $\psi$-pelletierine by the condensation of glutardialdehyde with methylamine and a derivative of acetone. 


\section{EXPERIMENAL.}

\section{Dipiperonylidenetropinone (Formula II).}

Potassium hydroxide (5 grams) dissolved in water (20 c.c.) was added to tropinone (2 grams) and piperonal (6 grams) dissolved in alcohol (100 c.c.), and the solution was then boiled under reflux during fifteen minutes. The derivative crystallised during the course of the reaction, and, after the addition of water, was collected, washed with water and alcohol, and crystallised from ethyl acetate. The yield was quantitative:

$$
\begin{gathered}
0.1139 \text { gave } 0.2991 \mathrm{CO}_{2} \text { and } 0.0543 \mathrm{H}_{2} \mathrm{O} . \mathrm{C}=71 \cdot 6 ; \mathrm{H}=5.3 . \\
\mathrm{C}_{24} \mathrm{H}_{21} \mathrm{O}_{5} \mathrm{~N} \text { requires } \mathrm{C}=71 \cdot 4 ; \mathrm{H}=5.2 \text { per cent. }
\end{gathered}
$$

Dipiperonylidenetropinone separates from ethyl acetate in bright yellow needles melting at $214^{\circ}$, and is sparingly soluble in most organic solvents. If it is rubbed on the side of a test-tube and sulphuric acid added, the substance acquires a coppery lustre and then passes into an intense royal-blue solution. This becomes green and finally yellow on dilution with water. The salts of the substance are in general sparingly soluble, but the acetate dissolves readily in water to a bright orange-yellow solution. Yellow, crystalline precipitates of corresponding salts are obtained on the addition to such a solution of hydrochloric, hydrobromic, nitric, sulphuric, oxalic, or picric acid. The hydrochloride crystallises from hot water, in which it is sparingly soluble, in yellow, microscopic needles. The substance is decomposed by hydroxylamine in boiling alcoholic solution with the formation of piperonaloxime, and presumably of the oxime of tropinone.

The formation of the dipiperonylidene derivative serves as a means of detection of the merest traces of tropinone, and the test may be applied in the following manner. A solution which it is suspected contains the base may first be acidified and evaporated in a vacuum in order to remove possible volatile neutral impurities, such as acetone. Alcohol, an excess of piperonal, and potassium hydroxide are then added, and the solution heated on the steam-bath for two or three minutes and then poured into ether. The ethereal solution is washed with water and separated, and then agitated with a little dilute hydrochloric acid. If tropinone is present, the hydrochloride of the derivative is so obtained in a crystalline condition. 
Synthesis of Tropinone from Succindialdehyde, Methylamine, and Acetone.

In this reaction, which has not yet been sufficiently investigated, it seemed that the tropinone could only be detected after the expiration of a brief period from the time of addition of the methylamine. If the reaction mixture was allowed to remain overnight, other basic ketones wers obtained which condensed with piperonal and interfered with the recognition of the desired product. Succindialdoxime ( 7 grams) suspended in water (30 c.c.) was converted into succindialdehyde by means of nitrous fumes generated from nitric acid (D 1.3) and arsenious oxide according to the method developed by Harries (Ber., 1901, 34, 1494). Water $(50$ c.c.) was then added, and the nitrous and nitric acids neutralised by means of an excess of precipitated calcium carbonate. After the addition of acetone (5 grams), and then methylamine ( 4.5 grams) dissolved in water (20 c.c.), the mixture was allowed to remain during half an hour. The solution was acidified with hydrochloric acid and tested for tropinone by the method described above. The small quantity of the crystalline hydrochloride was collected, treated with a little aqueous ammonia, and the base crystallised from ethyl acetate. The yellow needles melted at $214^{\circ}$, and at the same temperature when mixed with a specimen of dipiperonylidenetropinone. The colour reactions of the two specimens in concentrated sulphuric acid were identical.

\section{Synthesis of Tropinone from Succindialdehyde, Methylamine, and Ethyl Acetonedicarboxylate.}

$\alpha$-Succindialdehyde (1 gram) and ethyl acetonedicarboxylate $(2.5$ grams) were dissolved in alcohol $(20$ c.c.), and, after cooling in ice-water, a solution of methylamine $(0.5 \mathrm{gram})$ in alcohol (10 c.c.) was gradually added during an hour. After remaining overnight at the ordinary temperature, the alcohol was removed by distillation in a vacuum, and the residue boiled with dilute sulphuric acid for half an hour and then evaporated in a high vacuum to small bulk. Potassium hydroxide was then added in excess, and the mixture distilled in a current of steam. The distillate was exactly neutralised with hydrochloric acid, concentrated, and the tropinone contained was identified as in the former example by conversion into the dipiperonylidene derivative, as described above. In this case also the identity of the synthetical product was established by the method of mixed melting points. 


\section{Synthesis of Tropinone from Succindialdehyde, Methylamine, and Calcium Acetonedicarboxylate in Aqueous Solution.}

Succindialdoxime (3.5 grams) was converted into dialdehyde in the usual manner, and after the neutralisation with chalk the solution was made up to 40 c.c. and mixed with acetonedicarboxylic acid (4 grams) dissolved in water (20 c.c.) and neutralised by the addition of calcium carbonate. Methylamine (from 8 grams of the hydrochloride) in water (10 c.c.) was then gradually added, and the whole allowed to remain at the ordinary temperature during fifty hours. The liquid was filtered, acidified with hydrochloric acid, and concentrated in a vacuum, using a large flask on account of excessive frothing. The residue was then rendered alkaline with sodium hydroxide and distilled in a current of steam until the distillate was neutral to litmus. This solution was then acidified with hydrochloric acid, concentrated in a vacuum, and, after the addition of sodium hydroxide, repeatedly extracted with ether. The combined ethereal extracts were dried over potassium hydroxide, somewhat rapidly in order to avoid the formation of salts, and the solvent removed by distillation. The residue gradually crystallised, oily impurities were removed by contact with porous porcelain, and the substance was recrystallised from light petroleum (b. p. 50-60 $0^{\circ}$. Long, flattened, pointed, colourless needles were obtained melting at $42^{\circ}$, and at the same temperature when mixed with an equal quantity of tropinone which had been obtained by the oxidation of tropine.

In every respect the two specimens were identical. For further comparison the picrate was prepared. It crystallised from water in canary-yellow needles melting and decomposing at $218-220^{\circ}$ alone or when mixed with tropinone picrate, which had in this case been obtained by the oxidation of ecgonine.

The oil separated from the crystalline base was recovered from the plate by extraction with ether, and after removal of the solvent, piperonal, alcohol, and potassium hydroxide were added and the mixture heated during five minutes on the steam-bath. The precipitate obtained on the addition of water was collected, washed with alcohol, and then crystallised from ethyl acetate. Dipiperonylidenetropinone melting at $214^{\circ}$ was thus isolated.

In the above experiment there is some uncertainty as to the amount of succindialdehyde started from, since the author, no doubt through lack of practice, has never been able to obtain the high yield claimed by Harries of from 70 to 80 per cent. In order, therefore, to gain some idea of the yield of tropinone from succindialdehyde, the following experiment was made, but the 
conditions of the synthesis are still under investigation, and it is hoped that even better results may ultimately be obtained.

A solution of succindialdehyde containing inorganic salts was first prepared in the usual way and then distilled in a current of steam. The distillate was concentrated in a high vacuum until, as it later appeared, about a 10 per cent. aqueous solution was obtained. Ten c.c. of this solution were diluted with water, and the dialdehyde was converted into the diphenylhydrazone, as described by Harries (Ber., 1902, 35, 1188). The solution contained 0.96 gram of succinaldehyde. Twenty-five c.c. (containing 2.42 grams of the dialdehyde) were mixed with a solution of acetonedicarboxylic acid (6 grams) in water (75 c.c.) to which a sufficient excess of precipitated calcium carbonate had been added, and after cooling in ice-water, methylamine (3 grams) in water (10 c.c.) was gradually introduced. The reaction was allowed three days for completion, and the liquid was then acidified to Congo paper with hydrochloric acid and concentrated in a high vacuum. The residue was dissolved in alcohol and the tropinone converted into dipiperonylidene derivative in the usual manner. The product was collected, well washed with water and a little alcohol, and dried at $100^{\circ}$. It weighed $4 \cdot 1$ grams and was free from inorganic impurities. The substance was identified as dipiperonylidenetropinone, and the above data show that the yield of tropinone from succindialdehyde in this experiment was 42 per cent. of that theoretically possible.

The possibility that tropinone might result from the condensation of succindialdehyde or a derivative with acetone or a derivative and methylamine or by the addition of methylamine to a cycloheptadienone occurred simultaneously to Professor A. Lapworth and the author, and a joint research was contemplated, but Dr. Lapworth's preoccupation with more urgent investigations did not allow him to undertake this fresh experimental work. 